\title{
Dispersive measurements of superconducting qubit coherence with a fast, latching readout
}

\author{
I. Siddiqi, R. Vijay, M. Metcalfe, E. Boaknin, L. Frunzio, R.J. Schoelkopf, and M.H. Devoret \\ Departments of Applied Physics and Physics, Yale University, New Haven, Connecticut 06520-8284
}

\begin{abstract}
The "quantronium" is a superconducting qubit consisting of a split Cooper pair box in which a large tunnel junction is inserted. This circuit has a special bias point where the Larmor frequency is, to first order, insensitive to fluctuations in the bias parameters - the charge of the box island and the phase of the large junction. At this optimal working point, the state of the qubit can be determined by dispersive measurements that probe the second derivative of the state energy with respect to these bias parameters. We use the quantronium phase degree of freedom to perform a non-linear, dispersive measurement of its inductive response using bifurcation amplification. This novel readout projects the state of the qubit in a few nanoseconds, and its latching property allows us to record the resulting information in a few hundred nanoseconds. We have measured, using this technique, Rabi oscillations and Ramsey fringes with an improved signal to noise ratio and contrast. The speed of this new readout scheme also opens the door for a new class of experiments which would characterize the relaxation processes associated with the measurement protocol.
\end{abstract}

\section{INTRODUCTION}

Superconducting tunnel junction circuits were first proposed for quantum information processing several years ago, and at present, are the most advanced solid state qubits with the longest measured coherence times [1, 2, 3, 4, 5, 6, 7]. Yet, the physical origin of the noise sources limiting coherence are still debated, even though the theoretical formalism for treating the effects of noise in general is well developed [8, 9, 10]. It has been conjectured that impurities or defects found on-chip could act as such noise sources 11]. These parasitic elements may exist in the junction tunnel barriers, the metallic electrodes, the circuit substrate, or in some combination thereof. In addition, the shadow-mask evaporation technique used to fabricate many superconducting qubits typically generates extra electrodynamic resonators in close proximity to the qubit junctions [12]. These resonators can have a characteristic frequency comparable to the qubit Larmor frequency, and are thus suspected to decohere the qubit. The precise manner in which a qubit interacts with uncontrolled degrees of freedom in its environment depends on the topology of the tunnel junction circuit and how information is written to and read from the qubit. Circuits which have a high degree of symmetry can be significantly decoupled from a noisy environment [1, 13] when biased at special operating points. The choice of readout scheme is also highly significant. Dispersive measurements of the qubit state [14, 15] probe the reactive part of the response of the circuit, and are thus attractive since they minimally excite the spurious degrees of freedom described above.

We report the first coherence measurements of a superconducting qubit with a non-linear dispersive readout. Our approach involves coupling the "quantronium" qubit [1] to a novel superconducting amplifier - the Josephson bifurcation amplifier (JBA) [16]. The JBA is based on a non-linear electrodynamic resonator with two metastable oscillation states [17]. In order to perform a readout, the

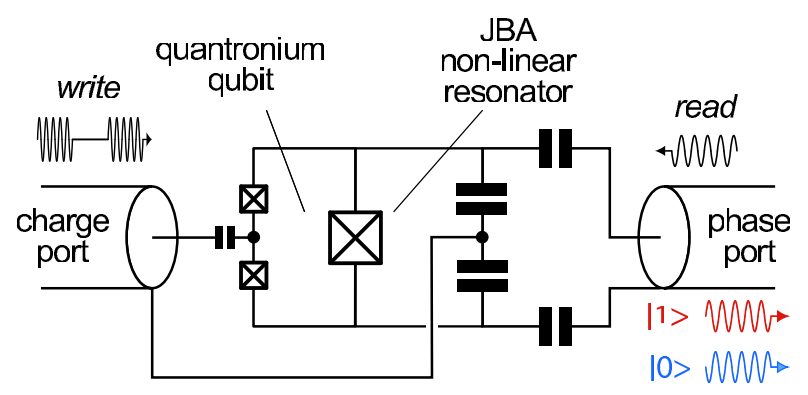

FIG. 1: Schematic of the measurement setup. The quantronium qubit is a split Cooper pair box with two small Josephson junctions in which a large junction is inserted for readout. This latter junction is shunted by two capacitors in series and forms the non-linear resonator of the JBA readout. The qubit state is manipulated by sending pulses to the charge port, while readout operation is performed by sending a pulse to the phase port and analyzing the phase of reflected signal, which carries information on the qubit state.

resonator is RF-energized to a level where its oscillation state now acts as a sensitive pointer of the qubit state. This technique does not generate any dissipation on chip since the resonator is only damped by circuitry outside the chip, i.e. a $50 \Omega$ transmission line with a matched circulator and amplifier, and enables a high-fidelity qubit readout with a $\mathrm{MHz}$ repetition rate. We have measured Rabi oscillations and Ramsey fringes with sufficient speed that real time filtering to correct for drifts in the charge and flux bias becomes possible. Also, several successive readouts may be performed within the energy relaxation time of the qubit $\left(T_{1}\right)$. This gives valuable information on the readout-induced interaction between the qubit and its environment, and accounts for the observed contrast. 


\section{THE HAMILTONIAN OF THE QUANTRONIUM QUBIT WITH JBA READOUT}

The principle of our experiment is schematically depicted in Fig. 1 and is based, as discussed above, on the quantronium qubit, a three junction circuit which is analogous to a one-dimensional atom. The set of three junctions consists of two small junctions, which we assume to be identical and have a Josephson energy comparable to the charging energy of the island between them, and a large junction, whose Josephson energy is approximately
100 times larger than that of each small junction. The gauge-invariant phase difference $\hat{\theta}$ of the island with respect to the mid-point of the capacitance shunting the large junction is analogous to the position of the electron relative to the nucleus of the atom, while the gaugeinvariant phase difference $\hat{\delta}$ across the large junction is the absolute position of the nucleus. Neglecting the dissipation induced in the transmission lines, the total hamiltonian of the split Cooper pair box with a JBA resonator is $\hat{H}(t)=\hat{H}_{b o x}(t)+\hat{H}_{r e s}(t)$ with

$$
\hat{H}_{b o x}(t)=4 E_{C}\left(\hat{N}-\frac{1}{2}+\frac{C_{g} U(t)}{2 e}\right)^{2}-\left(E_{J} \cos \frac{\hat{\delta}}{2}\right) \cos \hat{\theta} \quad ; \quad \hat{H}_{r e s}(t)=\frac{\hat{Q}^{2}}{2 C}-E_{J}^{R} \cos \hat{\delta}-\varphi_{0} I(t) \hat{\delta}
$$

Here, $\hat{N}$ and $\hat{Q} / 2 e$ are the momenta conjugate to the generalized positions $\hat{\theta}$ and $\hat{\delta}$, respectively. The constants $E_{C}, E_{J}, E_{J}^{R}, C$ and $C_{g}$ are the single electron charging energy of the island between the small junctions, the sum of the Josephson energy of the two small junctions, the large junction Josephson energy, the total capacitance shunting the large junction, and the gate capacitance, respectively. Here $\varphi_{0}=\hbar / 2 e$ is the reduced flux quantum. The control parameters $U(t)=U_{r f}(t) \cos \Omega t$ and $I(t)=I_{r f}(t) \cos \omega t$ are analogous to electromagnetic probe fields in an atomic system and induce a charge ex- citation of the write port and a phase excitation of the read port, respectively. This hamiltonian has been written supposing that the offset gate charge and loop flux have been compensated to operate at the optimal bias point where the charge $\frac{\partial \hat{H}}{\partial U}$ and the flux $\frac{\partial \hat{H}}{\partial I}$ have zero mean value in both the ground $|0\rangle$ and first excited $|1\rangle$ states of $\hat{H}_{b o x}$. Under these conditions, the qubit is minimally sensitive to charge and flux noise [1].

If we keep these two lowest states in the Hilbert space of the circuit we obtain an effective qubit hamiltonian

$$
\hat{H}_{e f f}=\frac{2 C_{g} U(t)}{e} E_{C} \sigma_{X}-\frac{E_{J}}{2} \sigma_{Z}+\hbar \omega_{p}\left(1+\lambda \sigma_{Z}\right) a^{\dagger} a-\mu\left(1+\frac{\lambda}{4} \sigma_{Z}\right)\left(a+a^{\dagger}\right)^{4}-f\left(a+a^{\dagger}\right) I(t)
$$

where

$$
\begin{aligned}
\omega_{p} & =\sqrt{\frac{E_{J}^{R}}{\varphi_{0}^{2} C}} \\
\lambda & =\frac{E_{J}}{4 E_{J}^{R}} \\
\mu & =\frac{E_{C}^{R}}{12}=\frac{1}{12} \frac{(e)^{2}}{2 C} \\
f & =\varphi_{0}\left(\frac{2 E_{C}^{R}}{E_{J}^{R}}\right)^{1 / 4}
\end{aligned}
$$

The photon annihilation operator $a$ is related to $\hat{\delta}$ by

$$
\hat{\delta}=\frac{a+a^{\dagger}}{\left(E_{J}^{R} / 2 E_{C}^{R}\right)^{1 / 4}}
$$

which represents the decomposition of the gaugeinvariant phase difference into annihilation and creation operators of the "plasma" mode whose bare frequency is $\omega_{p}$. The operators $\sigma_{X}$ and $\sigma_{Z}$ are the Pauli spin operators and $E_{C}^{R}$ is the single electron charging energy of the readout junction. In this effective hamiltonian, the expansion of $\cos \hat{\delta}$ is carried out only to the first anharmonic term, which describes the non-linear resonator dynamics with sufficient accuracy for a bifurcation readout.

Let us describe the role of each term in (1). The first term describes the influence on the qubit of the charge port drive which is used to manipulate its state. The second term is the Larmor term $\omega_{01}=E_{J} / \hbar$. We have supposed here that the ratio $E_{J} / E_{C}$ is sufficiently small that corrections to the Larmor frequency involving $E_{C}$ are small. To model the behavior of qubit samples with an appreciable $E_{J} / E_{C}$ ratio, we would keep higher order terms, yielding renormalized values of the coefficients in 
(1). The third term describes the dominant coupling between the qubit and the resonator. Note that this term commutes with the hamiltonian of the qubit when $U=0$, offering the possibility of quantum non-demolition measurements. The fourth term describes a decrease in the frequency of the resonator when its photon population increases 18. Finally, the fifth term describes the excitation of the resonator by the drive current applied through the phase port. When the drive current is increased while its frequency is sufficiently below $\omega_{p}$ the system becomes metastable with two possible dynamical states with different oscillation amplitudes, i.e. two possible photon populations [16]. We exploit this bistability for our readout, which we describe in the next section.

\section{QUBIT READOUT}

It is clear from the hamiltonian (1) above that the dynamics of the non-linear resonator depend on the value $\sigma_{Z}= \pm 1$ corresponding to the state of the qubit. In particular, the small oscillation "plasma" frequency $\omega_{p}^{\text {eff }}=$ $\omega_{p}(1 \pm \lambda)$ varies with the qubit state. We probe the nonlinear resonator by sending down the phase port transmission line a microwave pulse with carrier frequency $\omega=\omega_{p}-\Delta \omega$, such that the detuning $\Delta \omega>\frac{\sqrt{3}}{2 Q} \omega_{p}$ where $Q$ is the quality factor of the plasma resonance [17. In our circuit, the damping of the plasma resonance arises from the characteristic transmission line impedance $Z_{c}=50 \Omega$ and thus $Q=Z_{c} C \omega_{p} \simeq 10-20$. For this value of detuning, when ramping up the drive current $I_{r f}$ the resonator switches from one dynamical state to another when

$$
I_{r f}>I_{B}\left(\omega, \omega_{p}^{\mathrm{eff}}\right)
$$

where $I_{B}$ is the bifurcation current with expressions given in [19]. Therefore, by choosing the maximum pulse amplitude

$$
I_{B}\left[\omega, \omega_{p}(1-\lambda)\right]<I_{r f}^{\max }<I_{B}\left[\omega, \omega_{p}(1+\lambda)\right]
$$

we can determine, by measuring if the resonator has switched or not, whether the qubit was in state $|0\rangle$ or $|1\rangle$.

The dynamical states of the resonator differ in both the amplitude and phase of the forced oscillations at frequency $\omega$. In this work, we have chosen to use a reflectometry setup in which all the information about the resonator state is carried by the reflected drive signal phase $\phi$. This last property occurs because the probed circuit is not intrinsically dissipative (in absence of quasi-particles, which is very well realized in our measurements) and the power reflected from the chip is equal to the incident power in steady state. A further advantage of our non-linear resonator is that the switching is strongly hysteretic. Once a switching event has occurred we can decrease the drive current $I_{r f}$ to a value which, while much smaller than $I_{B}\left[\omega, \omega_{p}(1-\lambda)\right]$, is still higher than the reverse bifurcation "retrapping" current $I_{\bar{B}}$. This latching property conserves the information about the qubit state acquired during a small time interval $\tau_{m}$ in the resonator and allows us to probe the reflected phase $\phi$ during a time typically longer than $\tau_{m}$.

In Fig. 2, we present a typical histogram of the reflected drive signal phase $\phi$ corresponding to a drive current $I_{r f}$ which causes the resonator to switch, on average, half of the time. The histogram has 800,000 counts acquired in $200 \mathrm{~ms}$. For qubit measurements shown later, histograms with only 10,000 are used. The shape of the readout pulse used is schematically shown in the inset of Fig. 2. The rise time of the pulse is typically $20-40 \mathrm{~ns}$, the maximum current $I_{r f}^{\max }$ is applied for $40-120 \mathrm{~ns}$ and the latched section lasts $120 \mathrm{~ns}$, during which the recorded reflected signal phase $\phi$ is bimodal, with values differing by about $124^{\circ}$. We have chosen the phase reference so that the value $\phi=\phi_{\text {low }}=-62^{\circ}$ corresponds to the resonator in its initial state, while $\phi=\phi_{\text {high }}=62^{\circ}$ corresponds to the resonator having switched. We define the switching probability $P_{\text {switch }}\left(I_{r f}^{\max }, \Delta \omega,\left\langle\Psi\left|\sigma_{Z}\right| \Psi\right\rangle\right)$, where $|\Psi\rangle$ is the state of the qubit, as the weight of the histogram that lies above $\phi=\frac{\phi_{\text {low }}+\phi_{\text {high }}}{2}=0$.

\section{COHERENCE RESULTS}

We now present experimental results on two different qubit samples whose characteristic parameters are listed in Table I, along with a summary of our results. In the figures that follow, we only show data for sample A.

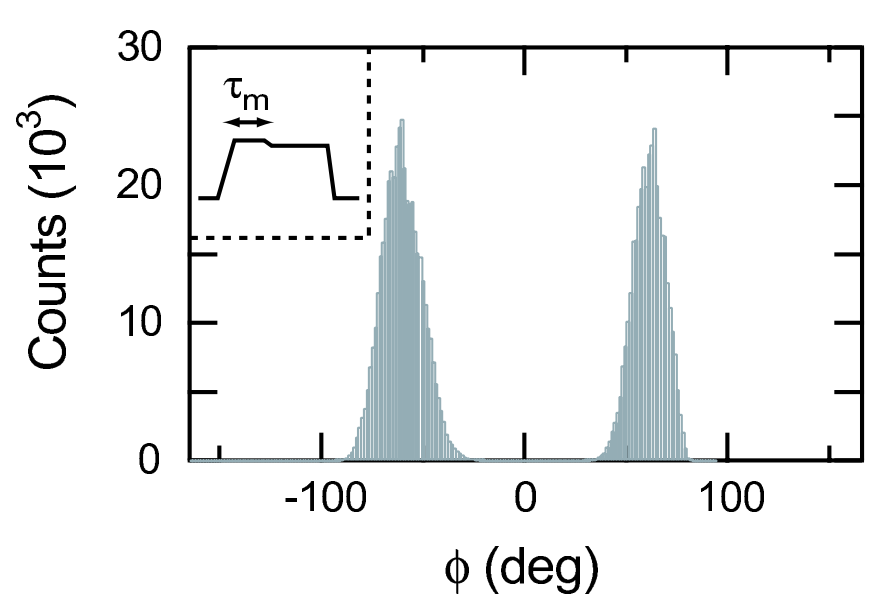

FIG. 2: Typical histogram of the phase of the reflected signal in the JBA readout when the maximum rf drive current is chosen so that the resonator switches approximately half of the time. The switching probability $P_{\text {switch }}$ is defined as the fraction of the histogram lying above $\phi=0$. The inset shows schematically the envelope of the readout pulse sent to the phase port. The qubit influences the switching probability during the time interval $\tau_{m}$ which here was $40 \mathrm{~ns}$. 
TABLE I: Parameters for two measured qubit samples. The readout frequency was $1.55 \mathrm{GHz}$ and $1.70 \mathrm{GHz}$ for samples A and B, respectively. The detuning was $6 \%$ of $\omega_{p}$. The parameter $\eta$ is the discrimination power of the readout.

\begin{tabular}{ccccccccc}
\hline \hline Sample & $\omega_{01} / 2 \pi(\mathrm{GHz})$ & $E_{J} / E_{C}$ & $T_{1, \text { typical }}(\mu \mathrm{s})$ & $T_{2}(\mathrm{~ns})$ & $T_{\text {echo }}(\mathrm{ns})$ & $\eta_{\exp }$ & $\eta_{\text {calc }}$ & $\eta_{\text {exp }} / \eta_{\text {calc }}$ \\
\hline $\mathrm{A}$ & 9.513 & 2.7 & 4.0 & 320 & $400-500$ & .48 & $.70 \pm .05$ & .69 \\
$\mathrm{~B}$ & 18.989 & 6.0 & 1.0 & 300 & 300 & .61 & $.70 \pm .05$ & .87 \\
\hline \hline
\end{tabular}

We first characterized our readout by measuring $P_{\text {switch }}$ as a function $I_{r f}^{\max }$ and $|\Psi\rangle$, as shown in Fig. 3 . The blue circles correspond to the qubit in its ground state, obtained by letting the qubit relax spontaneously, while the red circles correspond to the qubit in its first excited state obtained by applying a $\pi$ pulse, which will be discussed below. An important remark is that only a slight change in shape of $P_{\text {switch }}\left(I_{r f}^{\max }\right)$ between the two qubit states is observed, which indicates that the switching process itself does not contribute strongly to the relaxation of the qubit. In cases where the readout is suspected to induce significant relaxation, the switching probability curve for the qubit excited state displays a pronounced kink and can be obtained by a weighted average of the observed curve for the ground state and the prediction for the excited state $7,[20]$. The discrimination power of the qubit readout is defined as

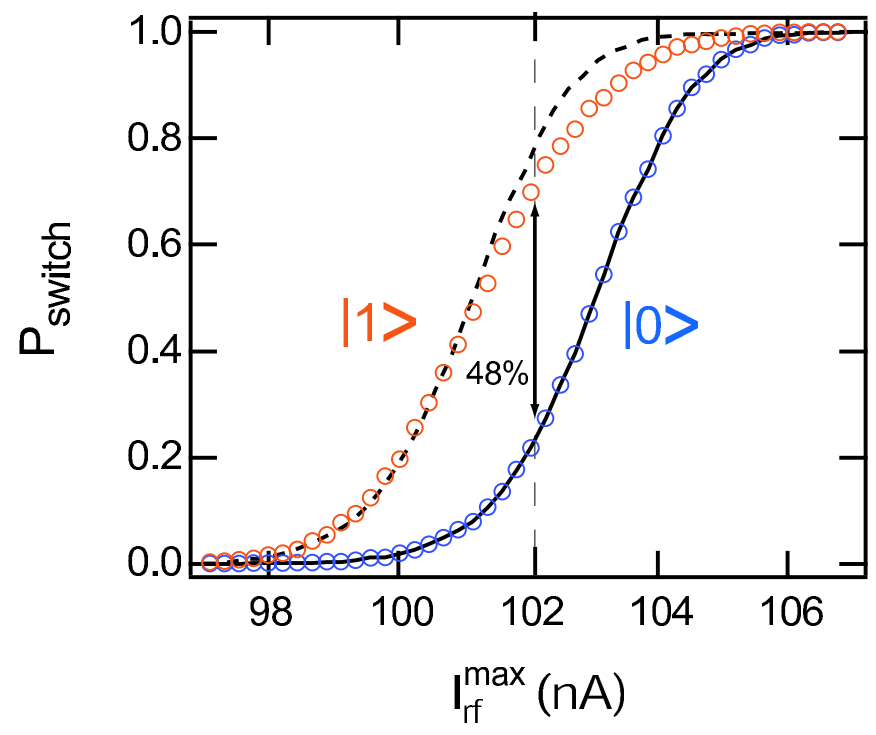

FIG. 3: Switching probability as a function of maximum drive current and qubit state for sample A. Dotted line represents value of drive current at which maximal discrimination power is observed. The width in current of the curves is in reasonable agreement with numerical simulations (data not shown). The solid line connects the observed data points in the $|0\rangle$ state and the dashed line is a copy of the solid line horizontally shifted to overlap the $|1\rangle$ state data at low values of $P_{\text {switch }}$.

$$
\eta=\max _{I_{r f}^{\max }}\left[P_{\mathrm{switch}}\left(\left\langle\sigma_{Z}\right\rangle_{\Psi}=1\right)-P_{\mathrm{switch}}\left(\left\langle\sigma_{Z}\right\rangle_{\Psi}=-1\right)\right]
$$

and its observed $\left(\eta_{\text {exp }}\right)$ and predicted $\left(\eta_{\text {calc }}\right)$ values are given in Table I. Numerical simulations 21] of the full circuit have been used to compute the predicted values of $\eta$. Note that several competing factors enter this calculation, yielding similar values for samples A and B. The error bars reflect uncertainties in the values of stray reactances on chip and the precise resonator temperature.

The observed discrimination power is about $15-30 \%$ smaller than expected, and we attribute this loss to spurious on-chip defects. In a set of experiments to be described in a later publication, we used two readout pulses in succession to determine that a $15-30 \%$ loss of qubit population occurs even before the resonator is energized to its operating point. As photons are injected into the resonator, the effective qubit frequency is lowered due to a Stark shift via the phase port 22]. When the Stark shifted frequency coincides with the frequency of an onchip defect, a relaxation of the qubit occurs. Typically, the qubit frequency spans $200-300 \mathrm{MHz}$ before the state of the qubit is registered by the readout, and $3-4$ spurious resonances are encountered in this range.

For future measurements, we have developed a new method to counter this effect. When applying a readout pulse via the phase port, we apply a compensating pulse via the charge port which Stark shifts the qubit to higher frequencies. When balancing these pulses, we have successfully reduced the net frequency shift to below $20 \mathrm{MHz}$ and have minimized population loss to defects before the resonator switches. To increase the expected discrimination power to unity, we must use samples with either a larger qubit $E_{J}$ or a stronger phase coupling between the qubit and readout resonator. The latter can be accomplished by using a resonator with two Josephson junctions in series.

Having characterized our readout discrimination power, we performed a series of experiments to assess the coherence of our qubit, namely the measurements of $T_{1}, T_{2}, T_{\text {echo }}$ and $\widetilde{T}_{2}$. These times characterize the decay of the excited state population after a $\pi$ pulse, the decay of Ramsey fringes, the decay of the echo signal after a $(\pi, \pi / 2, \pi)$ pulse sequence and the decay of the Rabi oscillations, respectively.

We first applied to the charge port a pulse at the Larmor frequency $\omega_{01}$ of varying duration $\tau$ and amplitude 

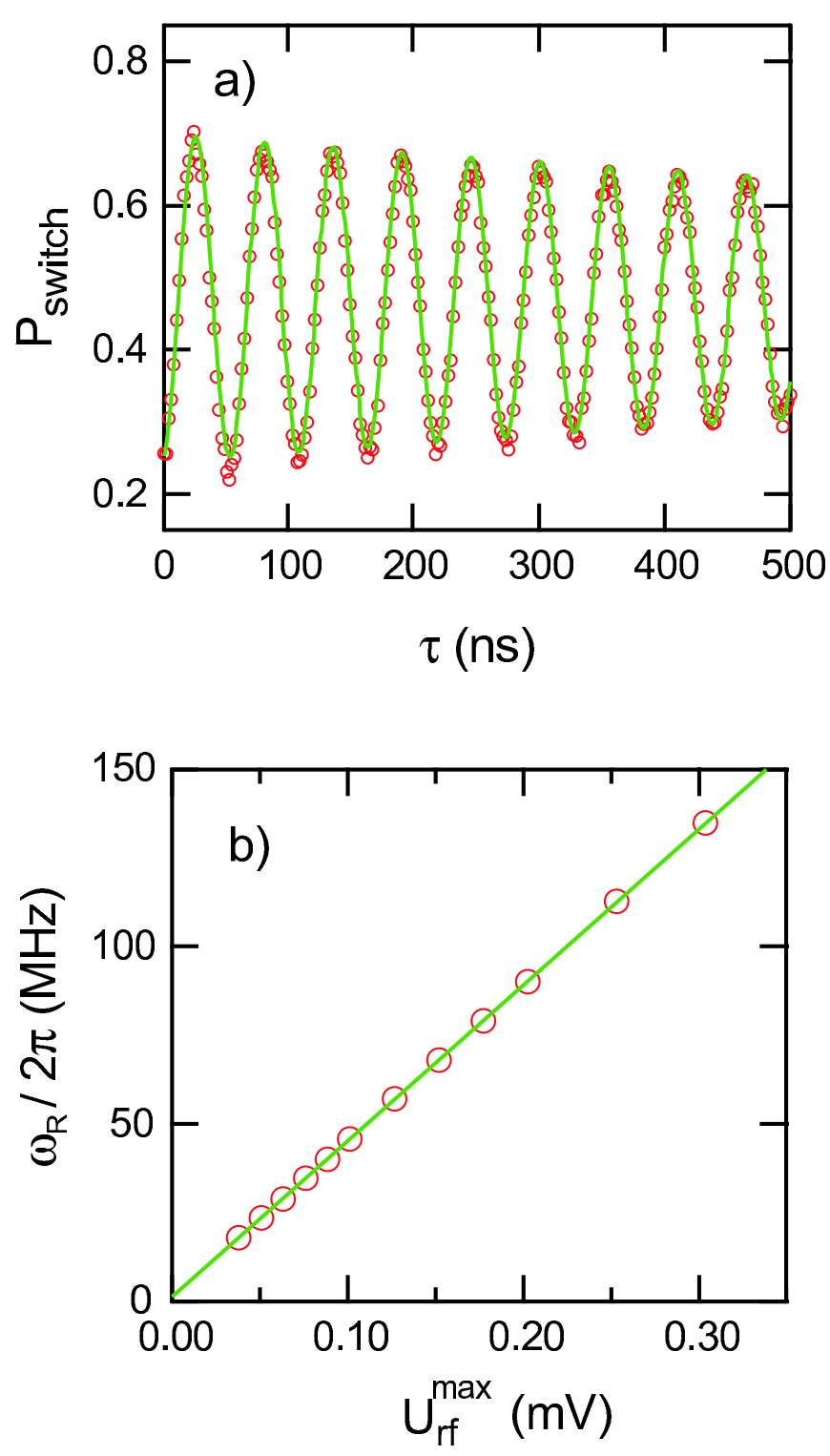

FIG. 4: a) Rabi oscillations of the switching probability of qubit sample $\mathrm{A}$ as a function of the duration $\tau$ of a square pulse applied on the gate with maximum amplitude $U_{r f}^{\max }=0.12 \mathrm{mV}$. Solid green curve is an exponentially decaying sinusoidal fit with $\widetilde{T}_{2}=1.6 \mu \mathrm{s}$. Total acquisition time is $3 \mathrm{mn}$ and duty cycle is $16 \mu \mathrm{s}$, set by $T_{1}$ (see below). b) Rabi oscillation frequency measured in (a) as a function of $U_{r f}^{\max }$. Green line is expected linear dependence.

$U_{r f}^{\max }$, which performs a rotation of the qubit about $\sigma_{X}$, followed by a readout pulse on the phase port. The resulting Rabi oscillations in the switching probability signal are plotted in Fig. 4a for varying $\tau$ and fixed $U_{r f}^{\max }$. Near $\tau=0$ we observe the $P_{\text {switch }}$ corresponding to qubit being in the $|0\rangle$ state. As the pulse length increases, $P_{\text {switch }}$ increases, goes through a maximum where the qubit is purely in the $|1\rangle$ state, defining at this point the length of a $\pi$ pulse. The switching probability then decreases back to the $|0\rangle$ state value, indicating a full $2 \pi$ rotation

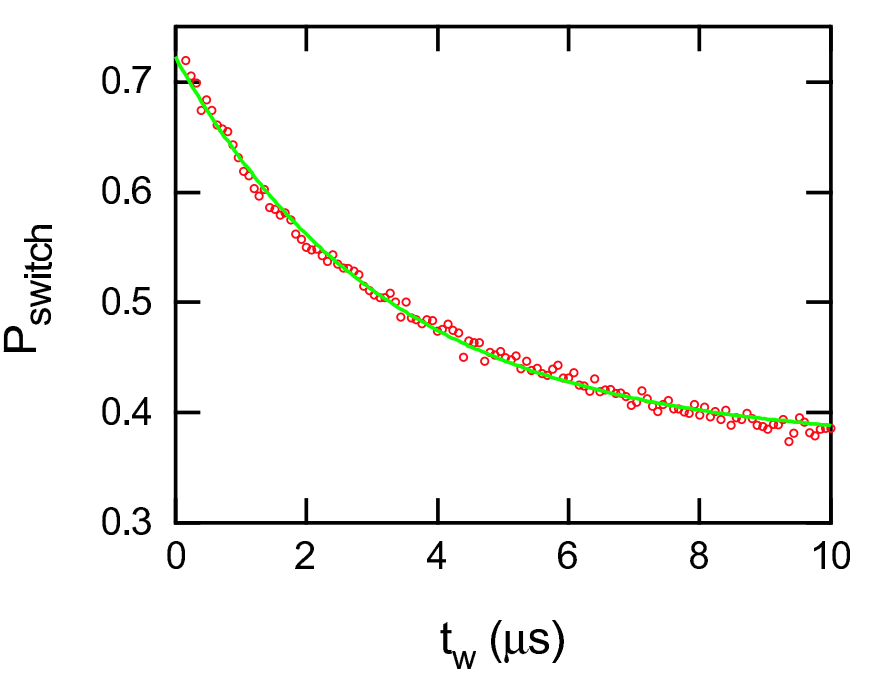

FIG. 5: Decay of the excited state switching probability after preparing the qubit in the excited state by a $\pi$ pulse, as a function of the waiting time $t_{w}$ between the preparation pulse and the readout pulse. Data for sample A. Solid green curve is an exponential fit with a $3.2 \mu$ s decay constant.

of the Bloch vector. This pattern repeats itself but with diminishing contrast. The decay time $\widetilde{T}_{2}$ is in the range $0.8-1.7 \mu$ s depending on the sample and precise biasing condition. The Rabi oscillation frequency $\omega_{R}$ is plotted as a function of $U_{r f}^{\max }$ in Fig. 4b. A linear dependence of $\omega_{R}$ with $U_{r f}^{\max }$ is observed, in agreement with theory. The shortest $\pi$ pulse we generated was $2 \mathrm{~ns}$ long, and was used in the echo experiments described below.

Having calibrated the $\pi$ pulse, we then performed a qubit energy relaxation measurement by introducing a waiting time $t_{w}$ between the $\pi$ pulse and the readout pulse. The decay of $P_{\text {switch }}$ with $t_{w}$, shown in Fig. 5, is well fitted by a single exponential, defining $T_{1}$. For sample A, $T_{1}$ was in the range $2.5-5 \mu \mathrm{s}$, and for sample $\mathrm{B}, T_{1}$ was between $1.0-1.3 \mu \mathrm{s}$. The values of $T_{1}$ obtained with our dispersive readout are comparable with the results of Vion et al. [1], and are significantly shorter than the values expected from coupling to a well thermalized $50 \Omega$ microwave environment shunting the qubit. The loss mechanisms giving rise to the observed energy relaxation are not understood at this time.

Following measurements of the qubit energy relaxation, we performed a Ramsey fringe experiment to determine the phase coherence of the qubit. In this experiment, two $\pi / 2$ pulses were applied to the charge port of the qubit at a frequency $10-20 \mathrm{MHz}$ detuned from $\omega_{01}$ followed by a readout pulse on the phase port. A free evolution time $\Delta t$ was introduced between the two $\pi / 2$ pulses. In Fig. $6, P_{\text {switch }}$ is plotted as a function of $\Delta t$. In the Ramsey sequence, the first $\pi / 2$ pulse tips the Bloch vector from the north pole to the equatorial plane. During the time $\Delta t$, the Bloch vector precesses around the equatorial plane and is then rotated again by the second $\pi / 2$ pulse. For $\Delta t=0$, the two $\pi / 2$ pulses back to 


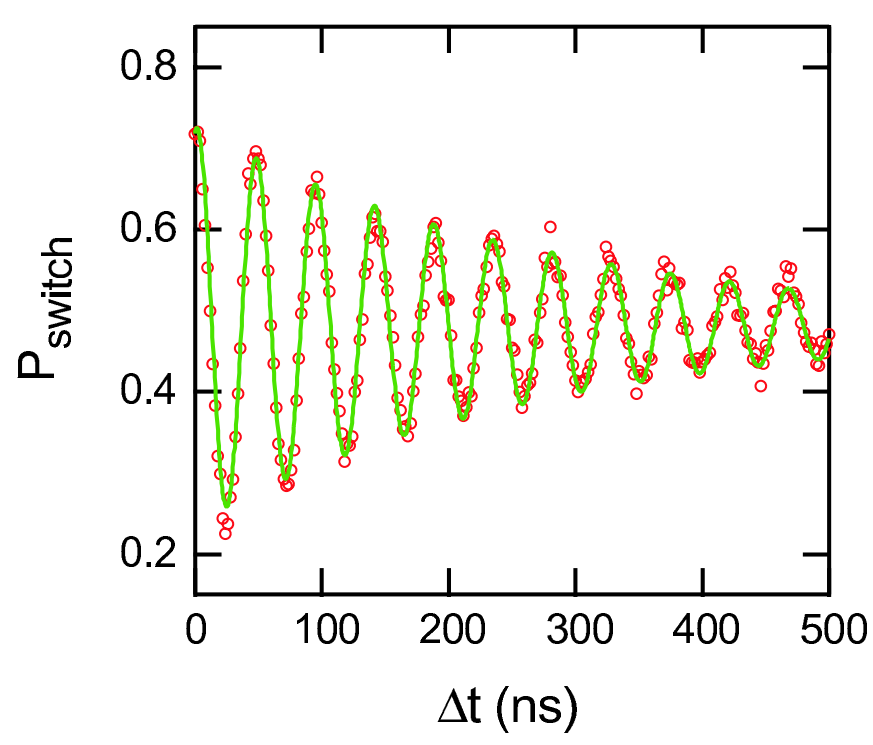

FIG. 6: Ramsey fringes obtained with two $\pi / 2$ pulses separated by the time interval $\Delta t$. The pulse frequency was detuned from the Larmor frequency by $20 \mathrm{MHz}$. The green curve is a exponentially decaying sinusoid fit. The decay time $T_{2}$ is 320ns. Same acquisition conditions as in Fig. 4.

back act as a single $\pi$ pulse and the observed value of $P_{\text {switch }}$ corresponds to the qubit being in the $|1\rangle$ state. As $\Delta t$ increases, $P_{\text {switch }}$ decreases until it reaches the value corresponding to the qubit being in the $|0\rangle$ state, corresponding to a free evolution time $\Delta t$ in which the Bloch vector makes a $\pi$ rotation in the equatorial plane. The switching probability then continues to increase for larger values of $\Delta t$ until it reaches a maximum value, corresponding to a time $\Delta t$ where the Bloch vector makes a full $2 \pi$ rotation in the equatorial plane. This oscillatory pattern then repeats but with decreasing contrast corresponding to the loss of phase coherence with time. The Ramsey fringes decay in a time $T_{2}$ which has a component due to energy relation and one due to pure dephasing: $1 / T_{2}=1 /\left(2 T_{1}\right)+1 / T_{\varphi}$, where $T_{\varphi}$ represents pure dephasing. In our measurements, $T_{2}$ is dominated by pure dephasing. For sample $\mathrm{A}, T_{2}=320 \mathrm{~ns}$, and for sample $\mathrm{B}, T_{2}=300 \mathrm{~ns}$.

In order to correct dephasing of the qubit due to low frequency noise 2, 23], we performed an echo experiment in which we inserted a $\pi$ pulse in the middle of the two $\pi / 2$ pulses of the Ramsey sequence. A set of Ramsey fringes and its corresponding echo decay are shown in Fig. 7 for sample A. For this sample, the decay constant was increased to $400-500 \mathrm{~ns}$ using the echo technique. For sample B, the echo technique did not increase the phase coherence time. We believe that for sample B, which has a large ratio of $E_{J} / E_{C}$ and is protected from $1 / f$ offset charge noise, the dominant source of dephasing is due to broadband noise emanating from residual photons in our readout resonator 24], thus explaining the inefficacy of the echo sequence. It is possible that the $50 \Omega$ environment shunting the qubit on the phase port side was

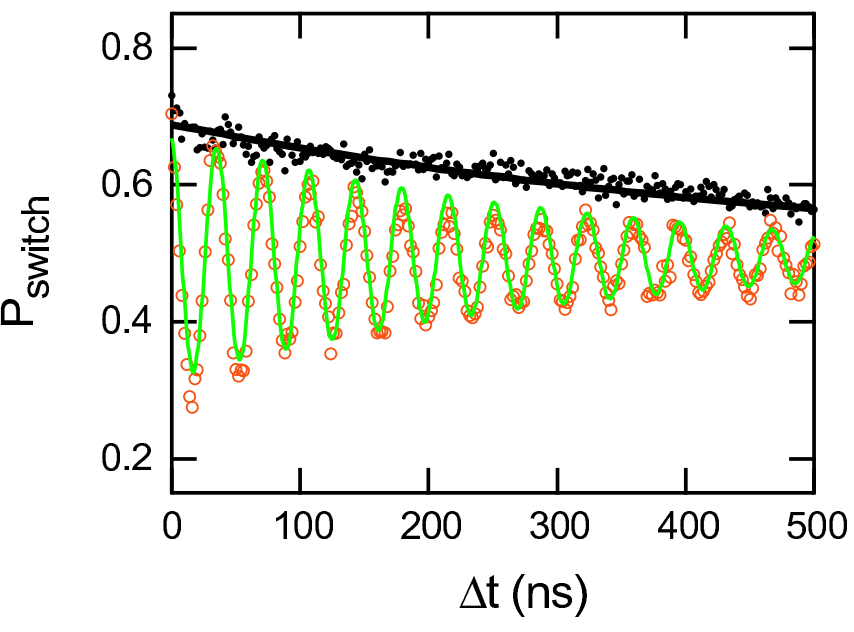

FIG. 7: Result of an echo experiment where a $\pi$ pulse was kept in the middle of the two $\pi / 2$ pulses separated by interval $\Delta t$ (black dots). The corresponding Ramsey fringe data is shown with red circles. The thick black curve is an exponentially decaying fit.

not fully thermalized to the refrigerator temperature of $10 \mathrm{mK}$. For sample A, where an improvement was observed with the echo sequence, there are likely two contributing factors. First, the ratio $E_{J} / E_{C}$ is much smaller and offset charge noise played a stronger role. The low frequency component of this noise can be corrected using an echo sequence. Second, we added more cryogenic attenuation in the transmission lines directly coupling to the phase port to reduce the resonator temperature, thereby potentially reducing the number of excess photons in the readout resonator and their associated dephasing.

\section{CONCLUSION}

In conclusion, we have successfully implemented a nonlinear dispersive readout of the quantronium qubit using the Josephson Bifurcation Amplifier. The readout speed and discrimination power show a significant improvement when compared with the DC switching readout used in the original quantronium measurements [1]. Perhaps even more important, in the present readout scheme, the total measurement time is much smaller than $T_{1}$, and it is possible to carry out experiments with multiple readout pulses to determine the information flow during a qubit readout and to account for any losses in qubit population. This important aspect can be used to determine the degree to which the measurement is quantum nondemolishing, and will be described in later publications.

The authors are grateful to Patrice Bertet, Daniel Esteve, Steve Girvin, Daniel Prober, and Denis Vion for helpful discussions. This work was supported by ARDA (ARO Grant No. DAAD19-02-1-0044), the Keck foundation and the NSF (Grant No. DMR-0325580). 
[1] D. Vion, A. Aassime, A. Cottet, P. Joyez, H. Pothier, C. Urbina, D. Esteve, and M.H. Devoret, Science 296, 886 (2002); E. Collin, G. Ithier, A. Aassime, P. Joyez, D. Vion, and D. Esteve, Phys. Rev. Lett. 93, 157005 (2004).

[2] I. Chiorescu, P. Bertet, K. Semba, Y. Nakamura, C. J. P. M. Harmans, J. E. Mooij, Nature 431, 159 (2004); P. Bertet, I. Chiorescu, G. Burkard, K. Semba, C. J. P. M. Harmans, D.P. DiVincenzo, J. E. Mooij, arXiv:cond-mat/0412485 v1 17 Dec 2004.

[3] T. Kutsuzawa, S. Saito, H. Tanaka, H. Nakano, K. Semba, and H. Takayanagi, arXiv:cond-mat/0501592 v1 25 Jan 2005.

[4] B.L.T. Plourde, T.L. Robertson, P.A. Reichardt, T. Hime, S. Linzen, C.-E. Wu, and John Clarke, arXiv:cond-mat/0501679 v2 29 Jan 2005.

[5] A. Wallraff, D. I. Schuster, A. Blais, L. Frunzio, J. Majer, S. M. Girvin, and R. J. Schoelkopf, arXiv:cond-mat/0502645 v1 27 Feb 2005, accepted for publication in Phys. Rev. Lett.

[6] R. McDermott, R. W. Simmonds, Matthias Steffen, K. B. Cooper, K. Cicak, K. D. Osborn, Seongshik Oh, D. P. Pappas and John M. Martinis, Science 307, 1299 (2005); John M. Martinis, K.B. Cooper, R. McDermott, Matthias Steffen, Markus Ansmann, K.D. Osborn, K. Cicak, Seongshik Oh, D.P. Pappas, R.W. Simmonds, and Clare C. Yu, arXiv:cond-mat/0507622 v1 26 Jul 2005.

[7] Y. Nakamura, private communication.

[8] D. J. Van Harlingen, T. L. Robertson, B. L. T. Plourde, P. A. Reichardt, T. A. Crane, and John Clarke, Phys. Rev. B 70, 064517 (2004).

[9] Alexander Shnirman, Gerd Schn, Ivar Martin, and Yuriy Makhlin, Phys. Rev. Lett. 94, 127002 (2005).

[10] G. Ithier, E. Collin, P. Joyez, P.J. Meeson, D. Vion, D. Esteve, F. Chiarello, A. Shnirman, Y. Makhlin, J. Schriefl, and G. Schon arXiv:cond-mat/0508588
[11] R. W. Simmonds, K. M. Lang, D. A. Hite, S. Nam, D. P. Pappas, and John M. Martinis, Phys. Rev. Lett. 93, 077003 (2004).

[12] D. Esteve, private communication.

[13] L. B. Ioffe, M. V. Feigel'man, A. Ioselevich, D. Ivanov, M. Troyer, G. Blatter, Nature 415, 503 (2002).

[14] A. Lupascu, C. J. M. Verwijs, R. N. Schouten, C. J. P. M. Harmans, and J. E. Mooij, Phys. Rev. Lett. 93, 177006 (2004).

[15] A. Wallraff, D. I. Schuster, A. Blais, L. Frunzio, R.S. Huang, J. Majer, S. Kumar, S. M. Girvin \& R. J. Schoelkopf, Nature 431, 162 (2004).

[16] I. Siddiqi, R. Vijay, F. Pierre, C.M. Wilson, M. Metcalfe, C. Rigetti, L. Frunzio, and M.H. Devoret, Phys. Rev. Lett. 93, 207002 (2004).

[17] M. I. Dykman and M. A. Krivoglaz, Physica A (Amsterdam) 104, 480 (1980).

[18] I. Siddiqi, R. Vijay, F. Pierre, C.M. Wilson, L. Frunzio, M. Metcalfe, C. Rigetti, R.J. Schoelkopf, and M.H. Devoret, Phys. Rev. Lett. 94, 027005 (2005).

[19] I. Siddiqi, R. Vijay, F. Pierre, C.M. Wilson, L. Frunzio, M. Metcalfe, C. Rigetti, and M.H. Devoret, arXiv:cond-mat/0507248 v1 11 Jul 2005.

[20] P. Bertet, I. Chiorescu, C. J. P. M. Harmans, and J. E. Mooij, arXiv:cond-mat/0405024 v1 3 May 2004.

[21] R. Vijay et al., in preparation.

[22] D. I. Schuster, A. Wallraff, A. Blais, L. Frunzio, R.-S. Huang, J. Majer, S. M. Girvin, R. J. Schoelkopf, Phys. Rev. Lett. 94, 123602 (2005).

[23] D. Vion, A. Aassime, A. Cottet, P. Joyez, H. Pothier, C. Urbina, D. Esteve, and M.H. Devoret, Fortschritte der Physik 51, 462 (2003).

[24] P. Bertet, I. Chiorescu, C.J.P.M. Harmans, and J. E. Mooij, arXiv:cond-mat/0507290 v1 13 Jul 2005. 